\title{
Use of the Wavelet Transform for Interference Detection and Mitigation in Global Navigation Satellite Systems
}

\author{
Luciano Musumeci and Fabio Dovis \\ Department of Electronics and Telecommunications, Politecnico di Torino, Corso Duca degli Abruzzi 24,10129 Turin, Italy \\ Correspondence should be addressed to Luciano Musumeci; luciano.musumeci@polito.it
}

Received 9 October 2013; Revised 20 December 2013; Accepted 30 December 2013; Published 26 February 2014

Academic Editor: Sandro Radicella

Copyright (C) 2014 L. Musumeci and F. Dovis. This is an open access article distributed under the Creative Commons Attribution License, which permits unrestricted use, distribution, and reproduction in any medium, provided the original work is properly cited.

\begin{abstract}
Radio frequency interference detection and mitigation are becoming of paramount importance due to the increasing number of services and applications based on the position obtained by means of Global Navigation Satellite Systems. A way to cope with such threats is the implementation in the receiver of advanced signal processing algorithm able to raise proper warning or improve the receiver performance. In this paper, we propose a method based on the Wavelet Transform able to split the useful signal from the interfering component in a transformed domain. The wavelet packet decomposition and proper statistical thresholds allow the algorithm to show very good performance in case of multiple pulse interference as well as in the case of narrowband interference, two scenarios in which traditional countermeasures might not be effective.
\end{abstract}

\section{Introduction}

Reliable positioning and navigation are becoming imperative in a growing number of applications that are being developed for public services and safety critical purposes. As a consequence, satellite and radio navigation is evolving in an accelerating pace and it is becoming a pervasive technology in a large number of consumer and professional devices. For such a reason, parallel to the development of techniques able to improve the positioning accuracy, the research is becoming of paramount importance to improve the robustness of the positioning methodologies in order to make sure that the navigation is trustworthy and the risks and threats are properly accounted for. As far as Global Navigation Satellite Systems (GNSS) based techniques, intentional or unintentional interference represents one of the main threats to be considered. GNSS receiver operation can be easily disrupted by interfering signal due to the extreme weakness of the GNSS signals reaching the GNSS user antenna. In fact, the presence of undesired RFI and other channel impairments can result in degraded navigation accuracy or complete loss of receiver tracking [1]. Furthermore, due to the lack of frequency allocations, the majority of the interference issues come from the presence of other communication systems which broadcast strong signals on frequencies within or located near the GNSS frequency bands. As an example, the terrestrial Digital Video Broadcasting (DVB-T) system may represent a real threat for the GNSS receiver operation. In fact, nonlinearity distortion generated in the DVB-T transmitter amplifier may lead to the generation of harmonics in the GPS L1 (and thus Galileo E1) frequency band.

In [2] a set of on-field experiments aiming at assessing the effect of DVB-T and VHF/UHF signals on GNSS receiver are described. Figure 1(a) shows the possible interference sources that can have secondary harmonics within the GPS L1, while Figure 1(b) specifies these potential interferers, with respect to the order of the harmonics. The DVB-T is transmitted within the frequency range of $174-230 \mathrm{MHz}$ (ultrahigh frequency, UHF band III) and 470-862 MHz (very high frequency, VHF bands IV and V). Additional details on DVB-T system impact on GNSS receiver performance can be found in [3].

Another example of out-of-band RF interference risk for the GNSS systems has been provided by the LightSquared case in the United States. The company deployed a groundbased 4G-LTE network that transmits on a frequency band right next to the primary GPS frequency (L1). Although LightSquared would have operated in its own authorized 


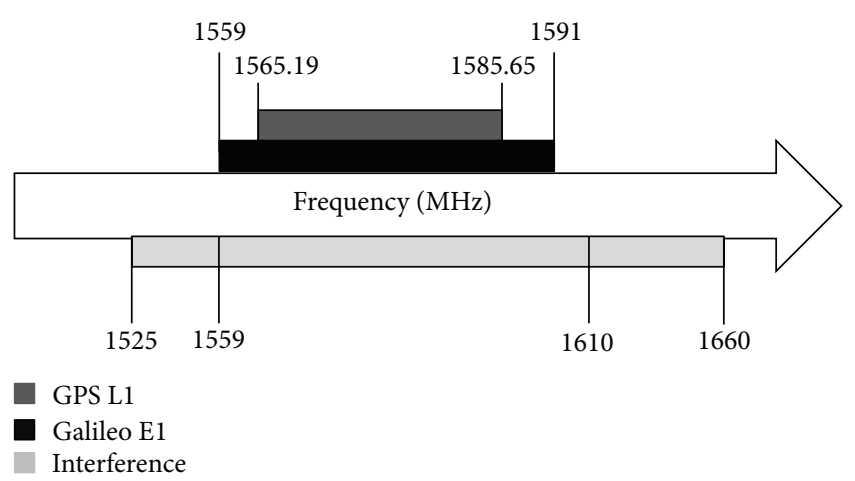

(a)
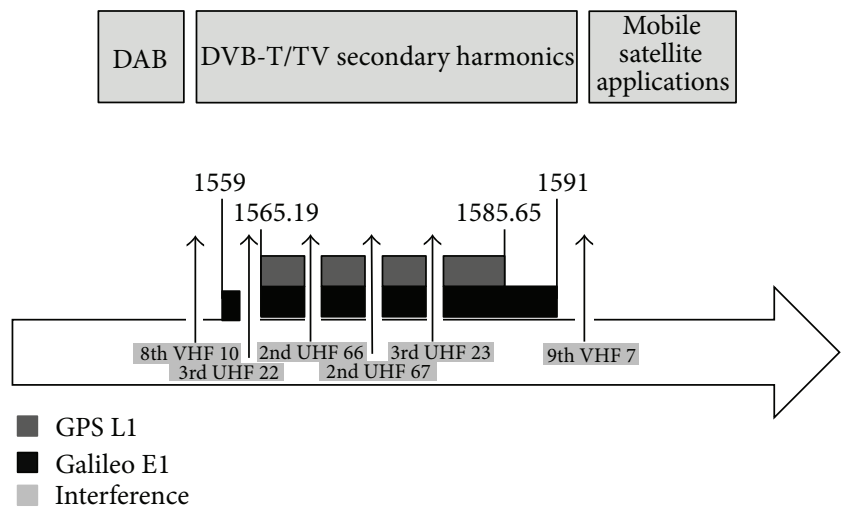

(b)

Figure 1: Possible in-band and out-of-band interferences (a). Secondary harmonics interference within the GPS L1 and Galileo E1 bands (b).

band, the proximity to the GPS signals induced overload or saturation of the radio frequency front-ends of the GNSS receivers. The threat to GPS was so strong that, following extensive testing and analysis, the Federal Communication Commission denied the LightSquared's terrestrial operations [4].

The interference issue is not only affecting the L1 band where the civil GPS signal and the Open Service of Galileo are broadcast. The future aidings for GNSS based aviation applications will be broadcast in the Galileo E5 and GPS L5 frequency bands which are shared with other Aeronautic Radio Navigation Systems, as described in [5]. In fact, GNSS based aviation aids will be broadcasted by the Space Based Augmentation Systems over the L5/E5 frequency band. However, such frequency bands are shared with other Aeronautical Radio Navigation Systems (ARNS) such as the Distance Measuring Equipment (DME) and the military one Tactical Air Navigation (TACAN). Both systems provide slant range information between an aircraft and a ground reference station, through the communication between two components, an interrogator installed on board of the aircraft and a transponder placed on the ground, usually within the airport area. Such DME/TACAN ground stations broadcast powerful modulated double-pulse signals which may corrupt the GNSS on-board receiver operation. A detailed description of such aeronautical systems is presented in [6]. It has been shown that on-board GNSS receiver flying at 40000 feet over Frankfurt airport area might be seriously threatened by DME/TACAN interference which may cause complete loss of lock of the GNSS signal.

Many other examples of communication systems which may represent a possible in-band unintentional interference sources for the GNSS are described in [3].

Last but not least, also intentional interference is a threat to be considered for civil GNSS community. Portable devices, jamming the GNSS bandwidth, typically broadcast signals frequency modulated where the instantaneous frequency sweeps a range of several $\mathrm{MHz}$ in a few microseconds affecting the entire GNSS band targeted by the device [7].

These few examples of interference generated by other communication systems proved that interference is a real issue in GNSS and that proper countermeasures have to be designed. In fact, the different interference sources may broadcast signals that are very different in terms of power, modulation, and pulse shape, thus making it difficult to have a universal countermeasure able to cope with all of them. In this paper, this issue is addressed working with a transformed domain approach that is able to deal with a large number of different kind of interfering sources, thus being effective in a wide range of scenarios.

The paper is organized as follows. Section 2 provides a model for the useful signal and the interference together with a basic interference signal classification. Section 3 will be devoted to a general description of the current state of the art of interference mitigation algorithms for GNSS. After introducing the Wavelet Transform in Section 4, an innovative interference mitigation algorithm based on the use of the wavelet packet decomposition will be presented in Section 5. Such an algorithm will be fully described, and its performance in detecting and suppressing interference will be discussed in Section 6.

\section{Signal Model and Interference Classification}

The received interfered GNSS signal at the receiver antenna can be written as

$$
s(t)=\sum_{l=0}^{L-1} y_{\mathrm{RF}, l}(t)+i(t)+\eta(t),
$$

where $L$ is the total number of GNSS useful signals, $y_{\mathrm{RF}, l}(t)$ is the useful GNSS signal received by the $l$ th satellite in line of sight, $i(t)$ is the additive interfering signal transmitted over a carrier frequency $f_{\text {int }}$ and characterized by a two-sided bandwidth $B_{\text {int }}$, and $\eta(t)$ is the additive white Gaussian noise. Before being fed to the acquisition and tracking block, the signal is first downconverted to an intermediate frequency, sampled, and quantized in the receiver front-end. Thus, the composite received signal at the ADC output according to [8] can be written as

$$
s_{\mathrm{IF}}[n]=s_{\mathrm{IF}}\left[n T_{s}\right]=Q_{k}^{u}\left[\sum_{l=0}^{L-1} y_{\mathrm{IF}, l}\left[n T_{s}\right]+i\left[n T_{s}\right]+\eta\left[n T_{s}\right]\right],
$$


where the function $Q_{k}^{u}$ denotes the quantization over $k$ bits, and $T_{s}$ is the sampling frequency. Expanding the term $y_{\mathrm{IF}, l}$, the expression for the single digitized GNSS signal affected by noise and interference components becomes (neglecting for the sake of simplicity the subscript $l$ )

$$
\begin{gathered}
s_{\mathrm{IF}}[n]=Q_{k}^{u}\left[\sqrt{2 C} d\left(n-n_{0}\right) c\left(n-n_{0}\right) \times \cos \left(2 \pi F_{D, 0} n+\phi_{0}\right)\right. \\
+i[n]+\eta[n]],
\end{gathered}
$$

where $C$ is the received GNSS signal power from one satellite in view, $d$ and $c$ are, respectively, the navigation data message content and the pseudorandom noise sequence, $F_{D, 0}=\left(f_{\mathrm{IF}}+\right.$ $\left.f_{0}\right) T_{s}$ is the Doppler affected frequency, $n_{0}=\tau_{0} / T_{s}$ is the digital code delay, and $\phi_{0}$ is the code delay.

$i[n]$ and $\eta[n]$ are the digitized interference and the digital Gaussian noise component, respectively. Given $B_{\mathrm{IF}}$ the frontend bandwidth, it can be shown that by sampling the signal at the Nyquist frequency $f_{s}=2 B_{\mathrm{IF}}$, the noise variance becomes

$$
\sigma_{\mathrm{IF}}^{2}=E\left\{\eta^{2}[n]\right\}=\frac{N_{0} f_{s}}{2}=N_{0} B_{\mathrm{IF}},
$$

where $N_{0} / 2$ is the power spectral density (PSD) of the noise.

A general classification of the interfering signals is based on their spectral characteristics such as their carrier frequency $f_{\text {int }}$ or their bandwidth $B_{\text {int }}$.

(i) Out-of-band interference refers to interfering signals whose carrier frequency is located near to the targeted GNSS frequency band $\left(f_{\text {int }}<f_{\mathrm{IF}}-B_{\mathrm{IF}} / 2\right.$ or $f_{\text {int }}>$ $\left.f_{\mathrm{IF}}+B_{\mathrm{IF}} / 2\right)$.

(ii) In-band interference refers to interfering signals with carrier frequency within the GNSS frequency band $\left(f_{\mathrm{IF}}-B_{\mathrm{IF}} / 2<f_{\mathrm{int}}<f_{\mathrm{IF}}+B_{\mathrm{IF}} / 2\right)$.

Moreover, interference can be further classified according to its characteristics in the frequency domain.

(i) Narrowband interference when the spectral occupation is smaller with respect to the GNSS signal bandwidth $\left(B_{\text {int }} \ll B_{\mathrm{IF}}\right)$.

(ii) Wide-band interference when the spectral occupation is comparable with respect to the GNSS signal bandwidth $\left(B_{\text {int }} \approx B_{\mathrm{IF}}\right)$.

(iii) Continuous wave (CW) interference which appears as a spike in the frequency domain $\left(B_{\text {int }} \rightarrow 0\right)$.

Furthermore, in general, interference might have timefrequency varying characteristics, for example, pulsed interference or chirp signals. The former is mainly characterized by on-off status of short duration (order of $\mu s$ ), which alternates in the time domain, whilst the latter is characterized by a linearly variation in time of the instantaneous frequency thus resulting in a wide-band interference. More details on interference classification can be found in [1].

\section{Interference Countermeasures}

Several kinds of interference countermeasures have been developed since the early years of GNSS. The methods proposed show advantages and limitations depending on the interfered scenario considered. In the following, a classification is provided in order to highlight the advantages and limitations of the different methods. Interference detection and mitigation techniques can be grouped in different families according to the point within the GNSS receiver chain they are applied on, as reported in Figure 2.

(i) Antenna level techniques which are based on the use of antenna arrays capable of generating radiation pattern which attenuates the interference signal coming from a determined direction [9].

(ii) Automatic Gain Control (AGC) level where the interference monitoring is performed detecting a persistent saturation status of the AGC (see, e.g., [10, 11]).

(iii) Postcorrelation techniques which are based on the analysis of the shape of the correlation function, in most cases exploiting a multicorrelator receiver [12].

(iv) Raw observable level techniques which are based on the processing of the raw samples at the Analog to Digital Converter (ADC) output.

In particular, among the interference countermeasures at raw observable level, it is possible to distinguish those which perform interference detection and cancellation in one single domain as the pulse blanking in the time domain or the notch filtering in the frequency domain. The innovative interference mitigation based on the use of the Wavelet Transform properties belongs to this latter family, since it performs detection and mitigation of the interference processing the received signal in a transformed domain.

Pulse blanking is the most traditional countermeasure suited for pulsed interference. Implemented by means of a digital circuit in the digital part of the receiver front-end, such technique performs an interference excision in the time domain by thresholding sample by sample the output of the Analog to Digital Converter (ADC) converter, as it is shown in Figure 3. Pulse detection in this case can be performed in either analog circuitry, through analog power measurement, or digital circuitry, looking at the histogram of the samples at the output of the ADC [13]. This simple mechanism performs pulsed interference suppression in the time domain and it offers good performance in the presence of pulsed interfering source characterized by a low Pulse Repetition Frequency (PRF). However, the technique shows limitation when highly dense in-time and strong interfering pulses are present since they lead the pulse blanking circuitry suppressing a large portion of the composite received signal and thus worsening the GNSS receiver performance due to the degraded quality of the received signal. In [6], it has been shown that, due to the described strong DME/TACAN interference environment, the pulse blanking of the receiver on-board suppresses about $56 \%$ of the incoming signal thus leading to the loss of lock of the weakest GNSS received signals.

Furthermore, the drawbacks of such simple interference countermeasure come from the fact that its performance is strongly dependent on the receiver front-end design. In fact, strong and dense in-time pulsed signals can cause the 


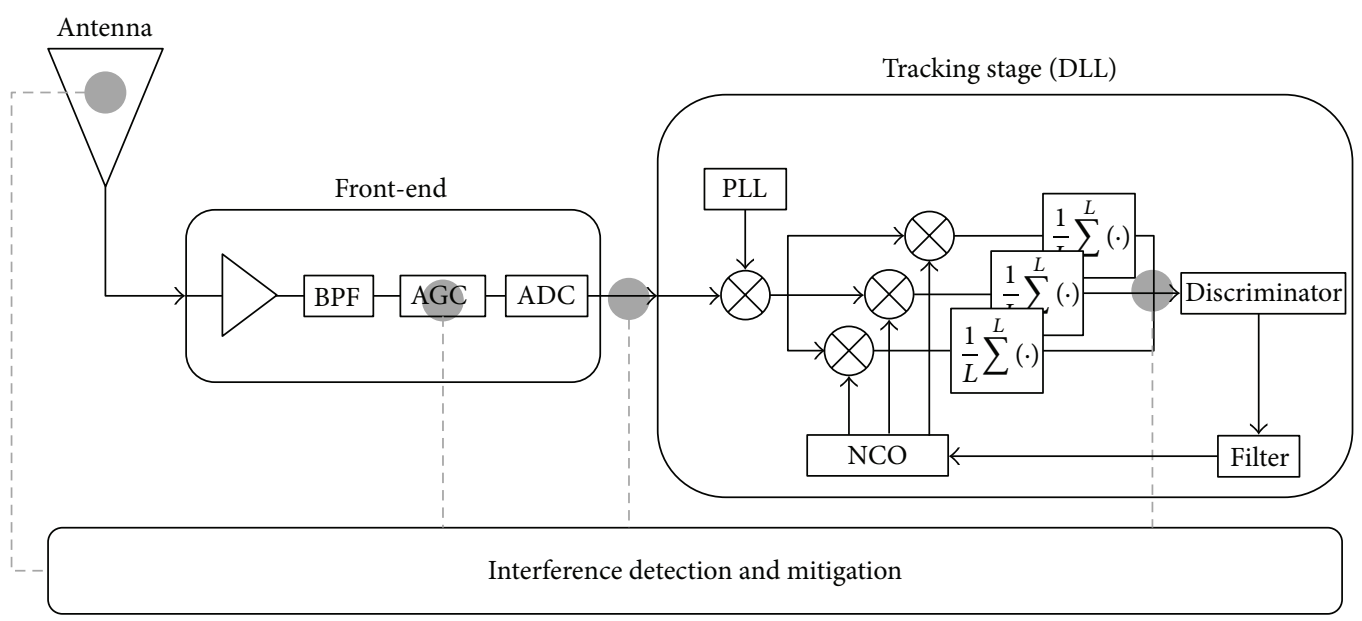

FIGURE 2: GNSS receiver chain block scheme.

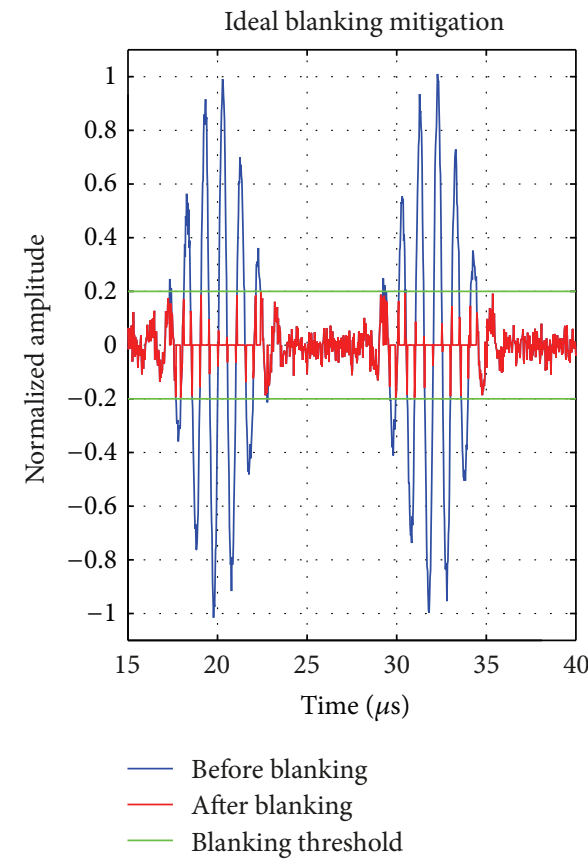

(a)

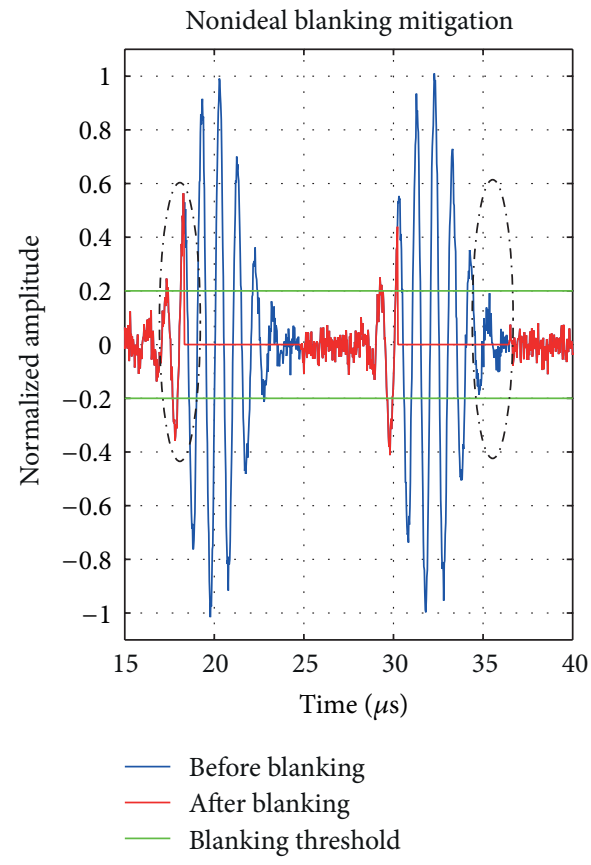

(b)

FIGURE 3: Signal before and after interference removal by means of ideal (a) and nonideal pulse blanking (b).

saturation of the active components in the GNSS receivers chain (e.g., amplifiers), which may require a recovery time to resume their normal state. This saturation effect may lead the pulse blanking suppressing the received signal even during the off-status of the pulse thus causing an increasing degradation on the received useful GNSS signal. In [14], it is mentioned that for a particular commercial receiver, an interference pulsed signal with a peak power $15 \mathrm{~dB}$ above the thermal noise is sufficient to saturate the last amplification stage of the receiver front-end. Furthermore, careful design of the Automatic Gain Control (AGC) is needed when a pulse blanker circuitry is present. A slow AGC sets the A/D input levels averaging the input signal power over a large time during which if too many pulses oscillations are present, the input dynamics of the ADC is not properly set [14]. The fact that the blanked samples should not be used for the AGC tuning in order to avoid ADC overloading has to be taken in consideration. In Figure 3, DME/TACAN pulse pair before (blue line) and after (red line) the application of both ideal (a) and nonideal (b) pulse blanking is shown. Nonideal blanking reveals a delay in pulse detection as well as a recovery time needed to the active components of the front-end to recover their normal operation, which produce a different mitigated signal. Furthermore, due to the bell shaping of the DME/TACAN and to the presence of the modulation over the pulse duration, not all the samples belonging to the pulse are suppressed leading to an increased noise floor [15, 16]. Unlike the pulse blanking, which works in the time domain, other 
interference countermeasures provide interference detection and cancellation in the frequency domain. As an example, interference cancellation can be performed suppressing the interference carrier frequency exploiting notch filtering. Such an interference countermeasure is well suited for CW or narrowband interference. In [16], notch filtering is proposed for suppressing DME/TACAN interference which appears in the frequency domain as Narrowband interference. In such a scenario the use of notch filters allows the suppression of the entire DME/TACAN pulse (tails included) but it reveals several drawbacks in the presence of multitone interference sources.

As an example, in [7], adaptive notch filtering scheme is described as interference countermeasure against jammers.

Another algorithm performing interference mitigation in frequency domain is the Frequency Domain Adaptive Filtering introduced in [17]. Such technique is based on a Fast Fourier Transform (FFT) of the samples at the ADC output. Interference suppression is performed through a sample by sample thresholding in the FFT domain. Basically, each FFT point is compared to a threshold, fixed according to a certain false alarm probability, and zeroed when the threshold is exceeded. Then an inverse FFT operation is performed on the manipulated incoming signal, in order to obtain back the signal in the time domain to feed to the acquisition and tracking blocks.

In most of the cases, interference is an unexpected and unpredictable event. Interference signal characteristics in time and frequency domain might be highly variable. For such a reason, research in this field is moving towards the investigation of innovative interference countermeasures able to represent the incoming signal in a two-dimensional domain, for example, the time-frequency domain, where interference component can be better isolated and removed without suppressing great portion of GNSS useful signal.

In [18], an interference mitigation algorithm based on the time-frequency representation of the GNSS received signal is proposed. Here, an Orthogonal-Like Gabor Expansion of the samples at the ADC output is employed in order to perform the time-frequency representation of the incoming interfered signal. Each value in the transformed domain is compared to a mask, which is computed through an Orthogonal-Like Gabor Expansion on the expected GNSS received signal that would be present in an interference-free environment. Such process allows to identify those coefficients in the time-frequency representation which represent interference information and that can be used to generate a synthetic reconstruction of the interfered signal. Interference suppression is performed subtracting a synthetic interference signal reconstruction from the original received signal. A drawback for this algorithm is that synchronization strategy is needed to perform a correct subtraction between the original signal and the synthetic reconstruction.

Recently, an innovative interference mitigation algorithm based on the use of the Wavelet Transform has been presented in $[19,20]$, for pulsed interference mitigation. Here, Wavelet Transform is employed to obtain time-scale representation of the incoming interfered signal. In the framework of the navigation satellite systems, the Wavelet Transform has been already extensively used; in fact, in literature, it is possible to find several research works in which the Wavelet Transform has been adopted mainly to cope with the multipath issue. In particular, in [21], a new trend extraction technique for multipath mitigation in carrier phase measurements domain using wavelet multiresolution analysis is presented. More detailed descriptions of this algorithm can be found in [22-25]. Multipath mitigation is not the only framework which Wavelet Transform has used. For instance, in [26], a singularity detection technique for GPS cycle slips based on the wavelet decomposition is described. In [27], an Empirical Mode Decomposition (EMD) exploiting the Wavelets' properties is described as a method to reduce the carrier phase measurements error. Finally in [28], methodology based on Wavelet Transform to evaluate the terrain and extract features along the vehicle path is presented. Those features which can be hazardous to a following vehicles path are of particular interest.

A different wavelet based mitigation algorithm approach for interference suppression will be presented and fully described in the next section.

\section{The Wavelet Transform}

Wavelet Transform overcomes the common limit of those transformations based on Fast Fourier Transform (FFT) as the Short Time Fourier Transform (STFT). The set of orthogonal basis functions which are employed for the STFT computation can be seen as bandpass filters having equal frequency bandwidths and thus representing a set of windows in time with equal duration. This leads to a different resolution in the characterization of high frequency phenomena with respect to the resolution achieved in characterizing low frequency phenomena. Exploiting fixed windows, many cycles of a high frequency signal can be captured, while for a low frequency signal very few cycles are within the windows. For such a reason, resolution of Fourier Transform is poor at low frequency, while it improves as the frequency increases [29]. Another drawback of using transformation based on fixed windows is revealed when considering the rising part of the signal. Very narrow window will help to localize the rising portion of the signal very well with respect to a wide window, at the expenses of a loss of information in the steady part of the signal, which is better characterized by wider windows. In order to overcome these issues, a set of functions which could better match the frequency components of the signal to be characterized is needed. From such basis functions, a filters bank where the low-pass filters response has narrower bandwidths (so wider in the time domain) than the high-pass filters response can be derived. Thus, a transformation based on windows which are functions of both time and frequency such that their bandwidths get narrower as the frequency decreases is needed. These requirements are accomplished by the basis functions used to perform the Wavelet Transform. In the STFT, all the bandpass filters are obtained by modulation of a single filter and thus they have equal bandwidth [29]. Concerning the Wavelet Transform, filters responses are obtained as

$$
h_{k}(t)=a^{-k / 2} h\left(a^{-k} t\right)
$$




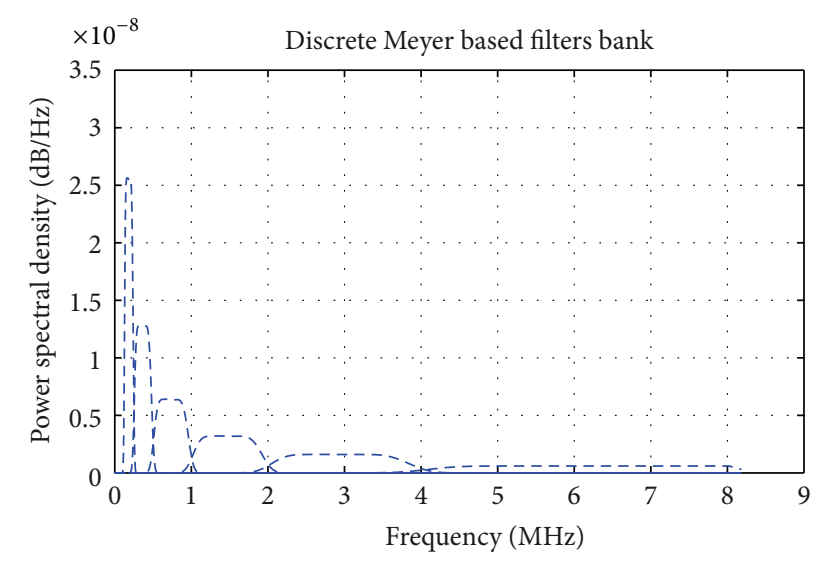

FIGURE 4: Meyer wavelet filters transfer functions.

or equivalently in the frequency domain

$$
H_{k}(j \Omega)=a^{k / 2} H\left(j a^{k} \Omega\right),
$$

where $a>1$ and $k \in \mathbb{Z}$.

As seen in (5) and (6), all the transfer functions are obtained by frequency-scaling operation of a prototype $H(j \Omega)$, the so-called mother wavelet, thus resulting in a nonuniform filters bank. As an example, Figure 4 shows the transfer functions of each branch of the nonuniform filters bank obtained by a dyadic scaling operation from the Meyer wavelet function. The scale factor $a^{-k / 2}$ is introduced as a normalization factor in order to ensure constant energy independent from $k$ as well as the ratio between the bandwidth and the center-frequency $\Omega_{k}$. Furthermore, since the filter bandwidth $H_{k}(j \Omega)$ is narrower for larger $k$, its output can be sampled at lower rate. Summarizing, given an arbitrary input $x(t)$, the output of the filter $h_{k}(t)$ is defined as

$$
\begin{aligned}
X_{\mathrm{DWT}}(k, n) & =\int_{-\infty}^{\infty} x(t) h_{k}(\tau-t) d t \\
& =a^{-k / 2} \int_{-\infty}^{\infty} x(t) h\left(a^{-k}(\tau-t)\right) d t .
\end{aligned}
$$

Replacing the continuous variable $\tau$ with $n a^{k} T$, it follows that

$$
\begin{aligned}
X_{\mathrm{DWT}}(k, n) & =a^{-k / 2} \int_{-\infty}^{\infty} x(t) h\left(n T-a^{-k} t\right) d t \\
& =\int_{-\infty}^{\infty} x(t) h_{k}\left(n a^{k} T-t\right) d t .
\end{aligned}
$$

The above integral represents the convolution between $x(t)$ and $h_{k}(t)$ evaluated at a discrete set of points $n a^{k} T$; that is, the convolution output is sampled with a spacing $a^{k} T$. The set of coefficients obtained for each value of $k$ provides the discrete Wavelet Transform. Thus, all the orthogonal basis functions composing the filters bank are derived by dilation $\left(t \rightarrow a^{-k} t\right)$ and shifting $\left(t \rightarrow t-n a^{k} T\right)$ of a prototype function $\psi(t)$, the mother wavelet; that is,

$$
\psi_{k n}(t)=a^{-k / 2} \psi\left(a^{-k} t-n T\right) .
$$

4.1. Discrete Time Wavelet Transform. It can be shown that a digital implementation of the Wavelet Transform can be equivalently implemented by using digital filters. The equivalent expression of (6) for digital filters would be

$$
H_{k}\left(e^{j \omega}\right)=H\left(e^{j 2^{k} \omega}\right) \longrightarrow H_{k}(z)=H\left(z^{2^{k}}\right),
$$

where $k$ is a nonnegative integer. In [29], it is shown that $H_{k}(z)$ is a multiband (rather than passband) filter; thus, in order to obtain passband filters, a low pass filter $G(z)$ is employed. In [30], $G(z)$ is defined as the mirror filter of $H(z)$ and together are called quadrature mirror filters. Thus, according to a dyadic scaling operation, the nonuniform filters bank responses are obtained as follows:

$$
H(z), G(z) H\left(z^{2}\right), G(z) G\left(z^{2}\right) H\left(z^{4}\right) \cdots .
$$

The Wavelet Transform can be extended to obtain the so-called Wavelet Packets Decomposition (WPD), where the discrete-time signal is passed through a uniform wavelet based filter bank, as shown in Figure 5. The scaling and shifting process is also iterated at higher frequencies, thus resulting in a uniform filter bank; the output of each is providing a set of coefficients (scales) each of which is representing a determined frequency portion of the incoming decomposed signal. Each stage of the uniform filters bank is composed by a filtering process through $H(z)$ and $G(z)$, respectively, the wavelet vector and the scaling vector individually shift orthogonal and orthogonal to each other, which produce a decomposition of the signal in high frequency component and low frequency component, followed by a downsampling operation.

\section{The Wavelet Based Mitigation Algorithm}

The proposed wavelet based mitigation algorithm is completely based on the WPD previously described. The algorithm for interference detection and suppression is mainly based on three steps.

(i) Decomposition phase where the incoming GNSS interfered signal is passed through the uniform filter bank thus achieving the time-scale representation. The number of wavelet stages to apply for the signal decomposition is a free parameter. Following in this paper, the optimal number of wavelet decomposition stages will be assessed with respect to the interference spectral characteristics and with respect to the GNSS receiver performance at both acquisition and tracking levels.

(ii) Detection-mitigation phase is performed in each scale obtained at the output of the filters bank. A simple blanking operation will be adopted in order to suppress those coefficients in each scale representing interference components. For such a reason, a criterion for the blanking threshold determination is needed. The adopted criterion is mainly based on a statistical characterization of the GNSS received signal at the ADC output. It is well known that 


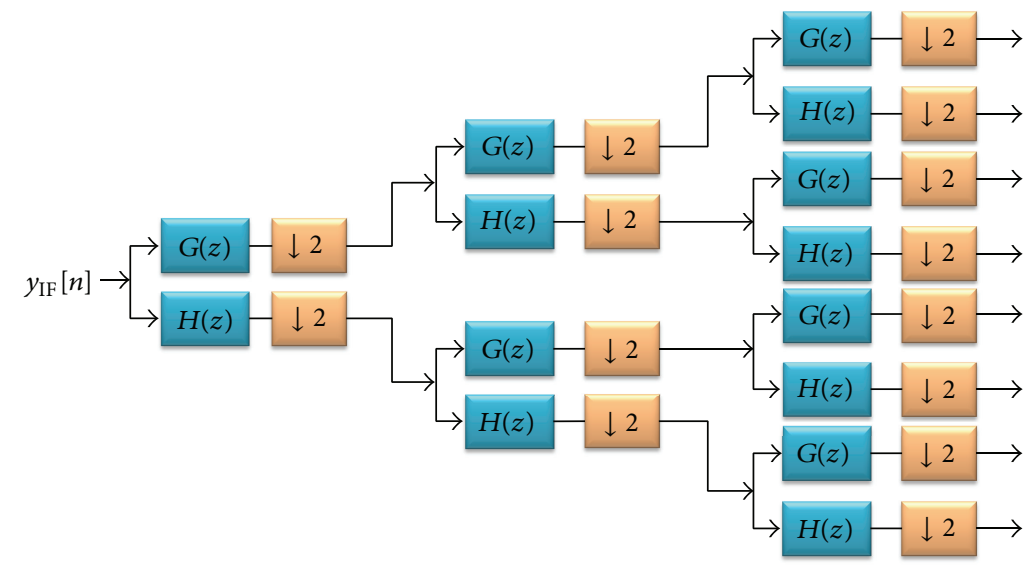

Figure 5: Wavelet Packet Decomposition by means of 3-stage uniform filters bank.

GNSS signal is completely buried in the noise at the user antenna level. Choosing a sampling frequency matching the Nyquist condition, the filtered digitized noise can be considered still uncorrelated; thus, it is allowed to assume that at the ADC output, the digitized GNSS signal in an interference-free environment is still Gaussian distributed with zero mean and variance $\sigma_{\mathrm{IF}}^{2}$. Denoting the false alarm probability $p_{f a}$ as the probability of the event in absence of interference, a generic sample at the ADC output crosses the blanking threshold $B_{\text {th }}$, it follows that

$$
p_{f a}=2 \cdot \int_{B_{\mathrm{th}}}^{\infty} \frac{1}{\sigma_{\mathrm{IF}}^{2} \sqrt{2 \pi}} e^{-x^{2} / 2 \sigma_{\mathrm{IF}}^{2}} .
$$

Thus, for a required false alarm probability $p_{f a}$, inverting (12), it follows that

$$
B_{\mathrm{th}}=\sigma_{\mathrm{IF}}^{2} \sqrt{2} \cdot \operatorname{erfc} c^{-1}\left(p_{f a}\right) .
$$

This blanking threshold magnitude is applied in each scale, since the wavelet filtering stages are performed with unitary energy filters.

(iii) Reconstruction phase achieved through an inverse Wavelet Packet transform applied on the wavelet scales after the mitigation phase. The main advantage of this algorithm with respect to the Gabor expansion based algorithm is that no signal storage for the signal decomposition as well as no synchronization operation at signal reconstruction is needed.

\section{Experimental Results}

This section will entirely focus on the application of such wavelet based interference mitigation algorithm in realistic interference scenarios. Analysis is performed exploiting software simulations. Wavelet based algorithm has been software implemented and offline applied on different data sets generated at intermediate frequency and representative of specific interference scenarios. Benefits in suppressing interference will be assessed looking at the time-frequency signal characteristics and comparing the signal quality before and after mitigation. A full software GNSS receiver, N-GENE [31], capable of processing Galileo and GPS signals over all the GNSS frequency bands, has been employed in order to assess receiver performance at both acquisition and tracking level after the wavelet based interference suppression. Such a software receiver is realized with FFT based acquisition scheme parallel in the time domain and tracking loops based on 2 nd order loop filters. Results in terms of separation between the correct acquisition peak in the search space and the noise floor $\left(\alpha_{\text {mean }}\right)$ as well as pseudorange tracking error and $C / N_{0}$ after the wavelet mitigation algorithm will be provided and compared with the receiver performance in an interference-free scenario.

Two macroscenarios have been considered.

(i) Pulsed interference scenario which is representative of a realistic interference scenario experienced by a GNSS receiver on board of an aircraft at 40000 feet over the central Europe region and the operation of which may be corrupted by the strong pulsed signals reaching the GNSS antenna and coming from the different ground DME/TACAN beacons.

(ii) Narrowband interference scenario generated with synthetic data at intermediate frequency. Different Narrowband interference scenarios will be taken into account considering different interference bandwidth and different off-set between the intermediate frequency and the interference carrier frequency. In this section, the best configuration of the parameters algorithm such as the number of wavelet decompositions or the filters length will be assessed looking at the receiver performance.

6.1. Pulsed Interference. An extremely realistic DME/TACAN interference scenario has been simulated through the use of the Interference Test Facility (ITF) available at the radio-navigation laboratory of the European Space Agency/European Space Research and Technology Centre 
(ESA/ESTEC). The ITF is a hardware software platform capable of generating a wide range of realistic interference scenarios and it is mostly devoted to the testing of GNSS hardware receiver performance under interference. More details on the different capabilities and configurations of this tool can be found in [32].

A realistic scenario of a GNSS receiver corrupted by the composite pulsed signal coming from up to $40 \mathrm{DME} / \mathrm{TACAN}$ stations broadcasting strong pulsed signals within the GPS L5 and Galileo E5a frequency bands has been simulated.

Figure 6 shows $10 \mathrm{~ms}$ of data collected at intermediate frequency $(9 \mathrm{MHz})$ sampled at $36 \mathrm{MHz}$. Spectral characteristics of the single DME/TACAN pulsed signal are shown in the plot on top. It appears as a narrowband interference with approximately $300 \mathrm{kHz}$ bandwidth. The entire spectrum is jammed due to the fact that several ground beacons have been simulated broadcasting pulses on different carrier frequency within the Galileo E5a and GPS L5 frequency bands. Under this condition, receiver operation is disrupted as demonstrated in [6].

$1 \mathrm{~ms}$ of coherent integration time combined with 80 noncoherent accumulations has been adopted in order to acquire the Galileo E5a pilot channel (PRN 20) in presence of strong DME/TACAN interference and results are shown in Figure 7. In this scenario, a high number of noncoherent accumulations have been employed in order to be able to detect the correct acquisition peak from the noise floor, leading to an acquisition metric $\alpha_{\text {mean }}$ equal to $24.8 \mathrm{~dB}$. Wavelet based mitigation algorithm has been applied to the interfered data-set. First, a time-scale representation of the signal at the ADC output is achieved exploiting 5 stages of a wavelet based filters bank, employing a Meyer wavelet family (see Figure 8).

After 5 stages of WPD, 32 scales are obtained, each of which represents a determined frequency component of the interfered received Galileo E5a signal. As it is shown in Figure 8, composite DME/TACAN signal reaching the user antenna is spread all over the time-scale domain. The black floors represent the blanking threshold applied for the interference component detection within each wavelet scale and computed according to a false alarm probability $p_{f a}$ of $10^{-4}$ as in (13). Once the time-scale representation of the incoming signal is achieved, an interference suppression algorithm based on a simple blanking operation is performed in each wavelet scale. Figure 9 shows the modified time-scale domain achieved after the blanking operation.

Such modified scales are fed to a wavelet based antitransformation block which is in charge of the signal reconstruction. Figure 10 provides a comparison between the time-spectral characteristics of the signal before and after the interference suppression through the WPD algorithm, showing the benefits of this algorithm when looking at the spectrum achieved after the mitigation (top plot). The effect of the pulsed interference is strongly attenuated.

Furthermore, unlike a common interference mitigation technique performed in the time domain, as the pulse blanking, where useful signal components are suppressed together with interference, the majority of the useful GNSS
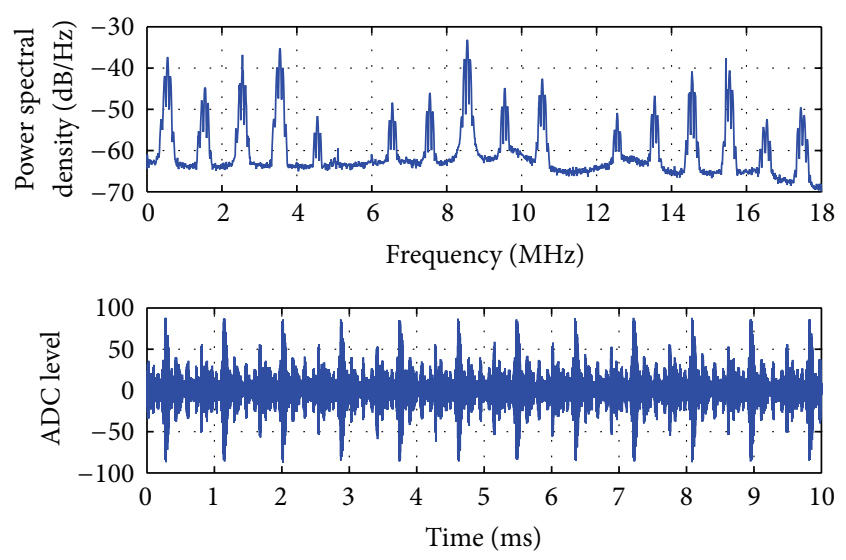

FIgURE 6: $10 \mathrm{~ms}$ of Galileo E5a signal at intermediate frequency affected by DME/TACAN pulsed interference.

Galileo E5aQ PRN20 search space: $\alpha_{\text {mean }}=24.8 \mathrm{~dB}$

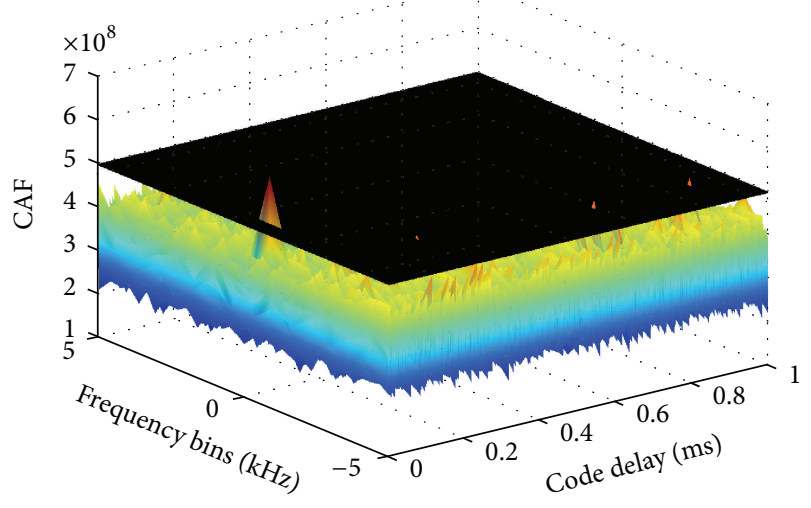

FIgURE 7: N-GENE acquisition search space in presence of DME/TACAN interference: Galileo E5a-Q channel, PRN 20.

signal power is saved, as confirmed by the absence of drops in the spectrum shown in Figure 10.

Figure 11 shows the acquisition search space obtained letting the software receiver acquire the data-set processed by the wavelet based mitigation engine. It can be clearly seen that acquisition of the Galileo E5a pilot channel (PRN 20) can be achieved already exploiting only 10 noncoherent accumulations combined with $1 \mathrm{~ms}$ of coherent acquisition time, thus reducing the Mean Acquisition Time (MAT) needed to detect the satellite. An excellent interference suppression is achieved as demonstrated by the high reduction of the noise floor in the search space. In fact, the separation between the main peak and the noise floor, denoted by $\alpha_{\text {mean }}$, is approximately $30.2 \mathrm{~dB}(4 \mathrm{~dB}$ higher than the interfered case in Figure 7) exploiting a considerably less number of noncoherent accumulations (only 10). Furthermore, interference suppression performed through this wavelet based method overperforms the interference mitigation performance achieved after applying a simple pulse blanking operation on the IF-samples of the collected data-set, as it can be seen in Figure 12, where, although correct Doppler and code delay acquisition 
Time-scale domain- $N=5$

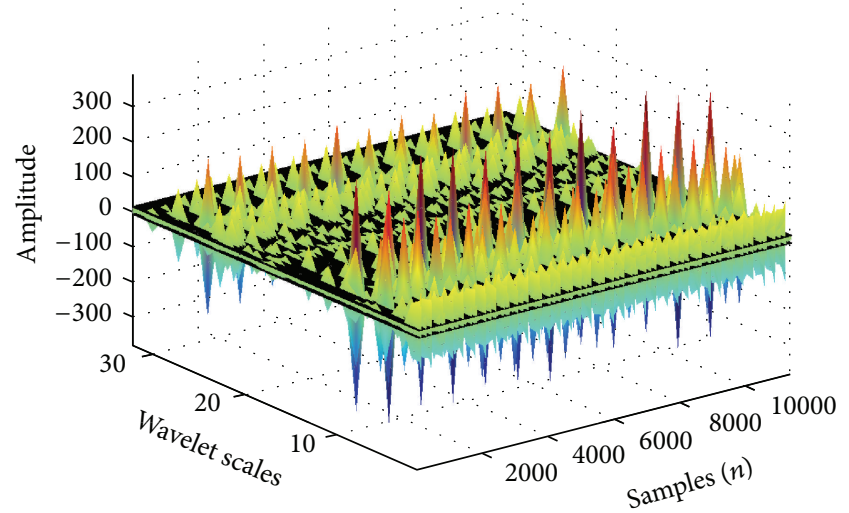

FIGURE 8: Time-scale representation achieved by 5 stages of wavelet packet decomposition.

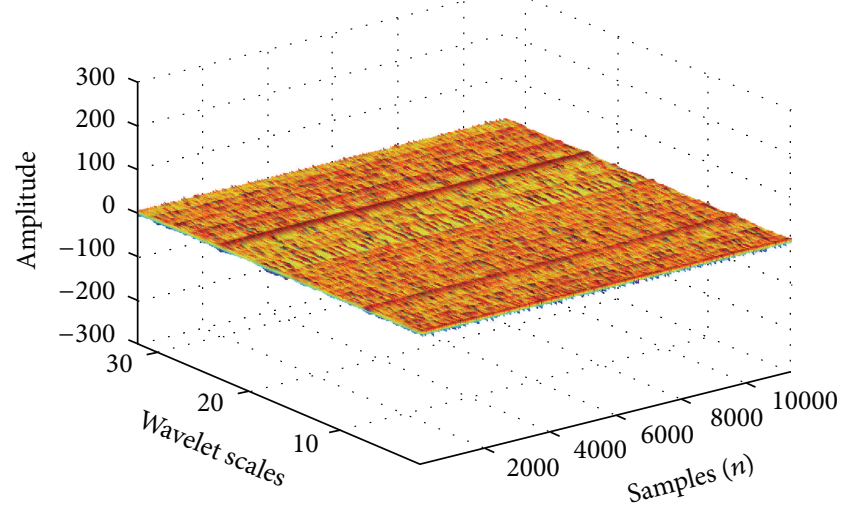

Figure 9: Time-scale after interference removal.
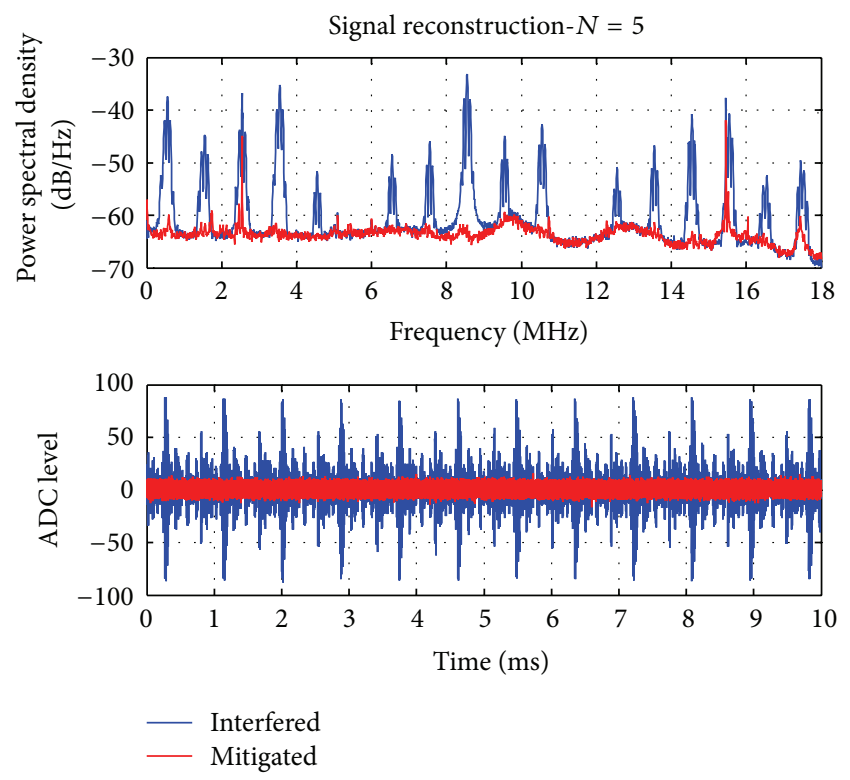

FIGURE 10: Galileo E5a signal at intermediate frequency before (blue curve) and after mitigation (red curve).
Galileo E5aQ PRN20 search space: $\alpha_{\text {mean }}=30.2 \mathrm{~dB}$

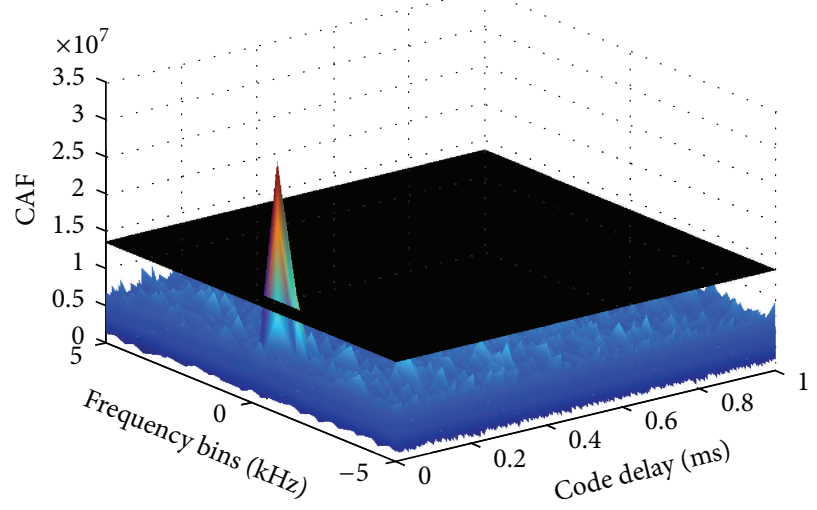

FIGURE 11: N-GENE acquisition search space after wavelet based mitigation algorithm: Galileo E5a-Q channel, PRN 20.

Galileo E5aQ PRN20 search space: $\alpha_{\text {mean }}=22.4 \mathrm{~dB}$

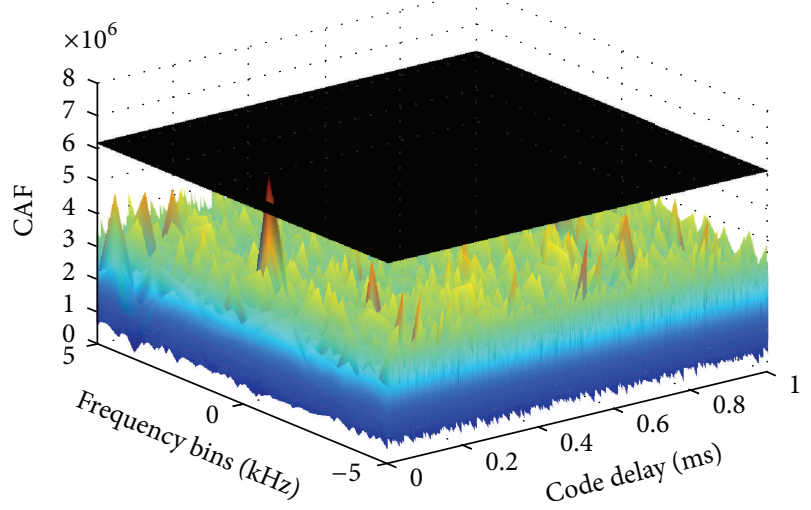

FIGURE 12: N-GENE acquisition search space after pulse blanking: Galileo E5a-Q channel, PRN 20.

is achieved, a noisier search space as well as a reduction of the correlation peak amplitude can be observed.

Concerning the software receiver tracking performance, carrier to noise density ratio $C / N_{0}$ and DLL jitter have been assessed. Figure 13 shows 10 seconds of the estimated $C / N_{0}$ during the Galileo E5a pilot channel tracking in presence of such a strong pulsed interference for three different scenarios:

(i) when no interference countermeasures are employed (red line),

(ii) when a traditional pulse blanking is employed (black line),

(iii) when the wavelet based interference mitigation algorithm is adopted (cyan line).

By means of the wavelet packet decomposition algorithm, a higher interference components suppression together with a negligible distortion of the useful GNSS signal components with respect to the case of the pulse blanking adoption is achieved, as demonstrated by higher average value of the estimated $C / N_{0}$ trend. The same conclusions can be drawn 


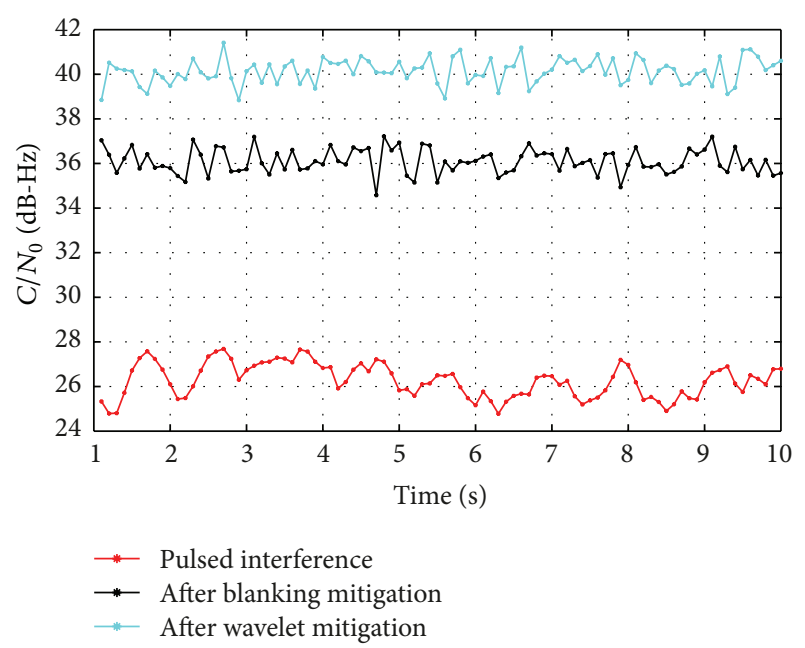

Figure 13: Carrier to Noise density ratio in absence of interference countermeasures (red), after pulse blanking (black), and after WPD based interference removal (blue).
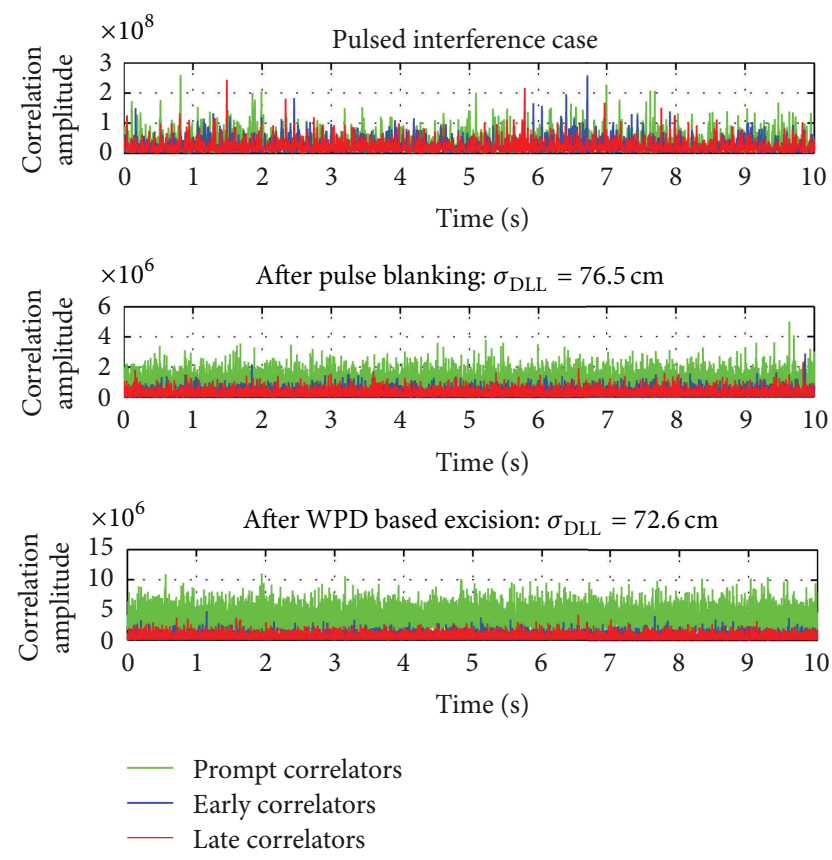

FIgURE 14: Early Prompt Late correlators during 10 seconds of Galileo E5a-Q channel PRN 20 tracking.

looking at the Early-Prompt-Late correlators values recorded during the tracking period for the three considered scenarios and depicted in Figure 14. We can clearly observe how the use of a traditional pulse blanking in presence of strong and dense in-time pulsed interference would cut off large portion of received signal thus leading to a decrease of the correlations amplitude and a minor separation between the E-L correlations amplitude with the prompt correlations amplitude and thus a higher DLL jitter output. Table 1 summarizes the $\mathrm{N}$-Gene acquisition and tracking performance for the considered scenarios.
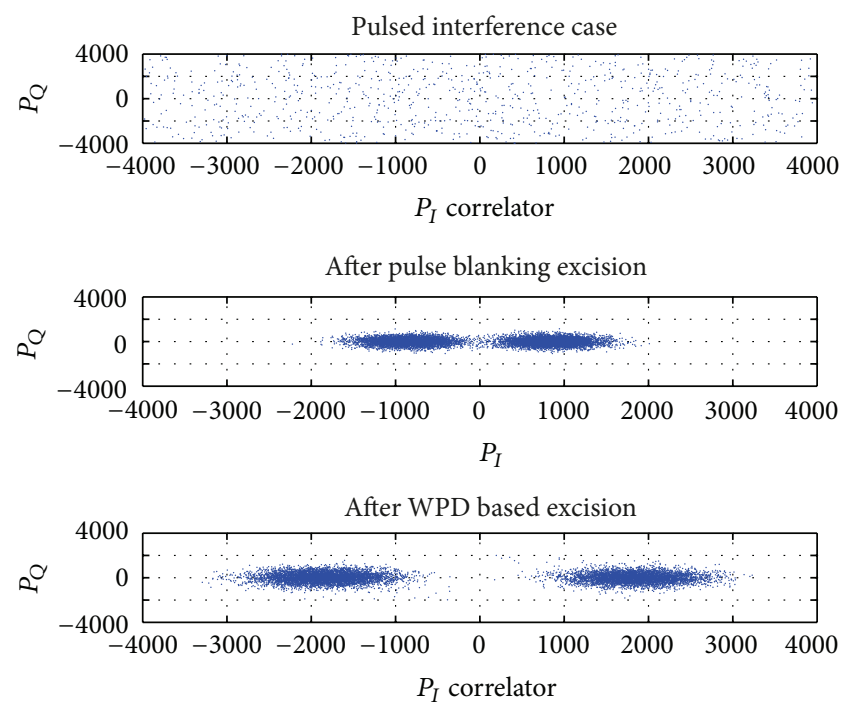

FIgURE 15: N-Gene data demodulation performance.

These results have been obtained setting a predetection integration time $T$ equal to $1 \mathrm{~ms}$ and choosing loops bandwidth equal to 5 and $15 \mathrm{~Hz}$, respectively, for the DLL and PLL.

Finally, data demodulation performance can be observed in Figure 15, where the prompt in-phase and quadrature correlations are plotted in the complex plane. As expected, tracking and thus correct data demodulation can not be achieved if no interference countermeasures are employed (top plot), while the data demodulation is noisier when employing the pulse blanking for interference suppression with respect to the case when the WPD algorithm is used.

Such results at tracking level confirm the capability of this wavelet based mitigation algorithm in effectively suppressing interference and saving useful GNSS signal components.

6.2. Narrowband Interference. This section is devoted to the performance analysis of the wavelet based mitigation algorithm in mitigating Narrowband interference. The analysis addresses the problem of finding the best trade-off between the choice of the wavelet based mitigation technique parameters such as number of wavelet decomposition stages $N$ and its computational burden. Such trade-off analysis is correlated with the Narrowband interference spectral characteristics. A digital GNSS signal generator [33] has been adopted in order to generate synthetic GPS L1 data combined with Narrowband interference. Several Narrowband interference scenarios have been considered, and a parametric study with respect to interference bandwidth $B_{\text {int }}$, interference carrier frequency $f_{\text {int }}$, and number of wavelet decomposition stages $N$ has been performed. In order to find the best tuning of the WPD based algorithm parameters, its application on synthetic interfered GNSS data has been performed and performance in suppressing interference has been assessed. The results which will be presented in the following paragraphs have been preliminarily discussed in [34].

6.2.1. Performance with respect to the Wavelet Decomposition Depth $N$. The first analysis has been devoted to assess the 
TABLE 1: Acquisition and tracking performance comparison.

\begin{tabular}{lcccc}
\hline Scenario & Noncoherent accumulations $K$ & $\alpha_{\text {mean }}(\mathrm{dB})$ & $C / N_{0}(\mathrm{~dB}-\mathrm{Hz})$ & - \\
\hline DME/TACAN interfered & 80 & 24.8 & $\sigma_{\mathrm{DLL}}(\mathrm{cm})$ \\
After pulse blanking mitigation & 10 & 22.4 & - & 36 \\
After WPD based mitigation & 10 & 30.2 & 40.5 & 72.6 \\
\hline
\end{tabular}

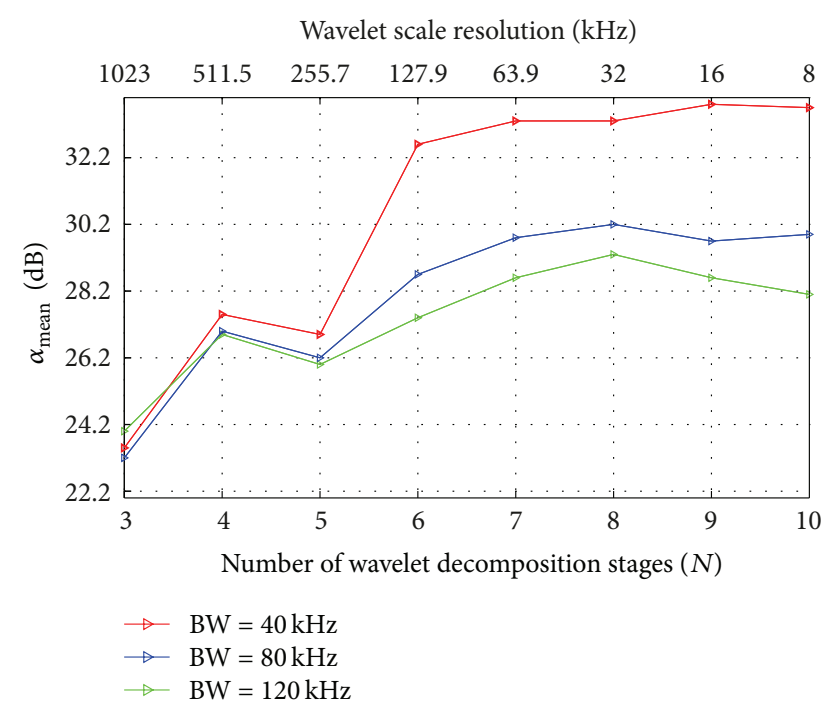

FIgURE 16: Acquisition metric $\alpha_{\text {mean }}$ versus the WPD stages $N$ for different interference bandwidth.

impact of the number of wavelet decomposition stages on the Narrowband interference suppression performance. Different interference scenarios have been considered, combining GPS L1 C/A code signals with Narrowband interference $200 \mathrm{kHz}$ far from the intermediate frequency. Results are shown in Figure 16, where the trend of the acquisition metric $\alpha_{\text {mean }}$ is plot versus the number $N$ of decomposition stages. Acquisition performance is achieved using $1 \mathrm{~ms}$ of coherent integration time and 20 noncoherent accumulations. The three lines referred to three different interference scenarios characterized by the presence of Narrowband interference with, respectively, 40, 80, and $120 \mathrm{kHz}$ of bandwidth. Increasing the acquisition stage increases the wavelet scale resolution and thus its frequency selectivity. In all the three interference scenarios, for increasing values of $N$, the WPD based algorithm provides better performance in capturing and isolating narrowband interference, thus leading to better interference suppression with a limited removal of useful signal components, as demonstrated by the increasing trend of $\alpha_{\text {mean }}$ in Figure 16. However, a saturation effect can be observed for higher value of $N$ (greater than 7). In such a region, acquisition performance is not anymore improving since wavelet scale resolution is already comparable or narrower with respect to the interference bandwidth. Moreover, as expected, performance of such technique is limited by interference bandwidth. At higher interference bandwidth, lower acquisition metric values are achieved.

6.2.2. Wavelet Families Comparison and Interference Carrier Offset. So far, time-scale representation of the signal at

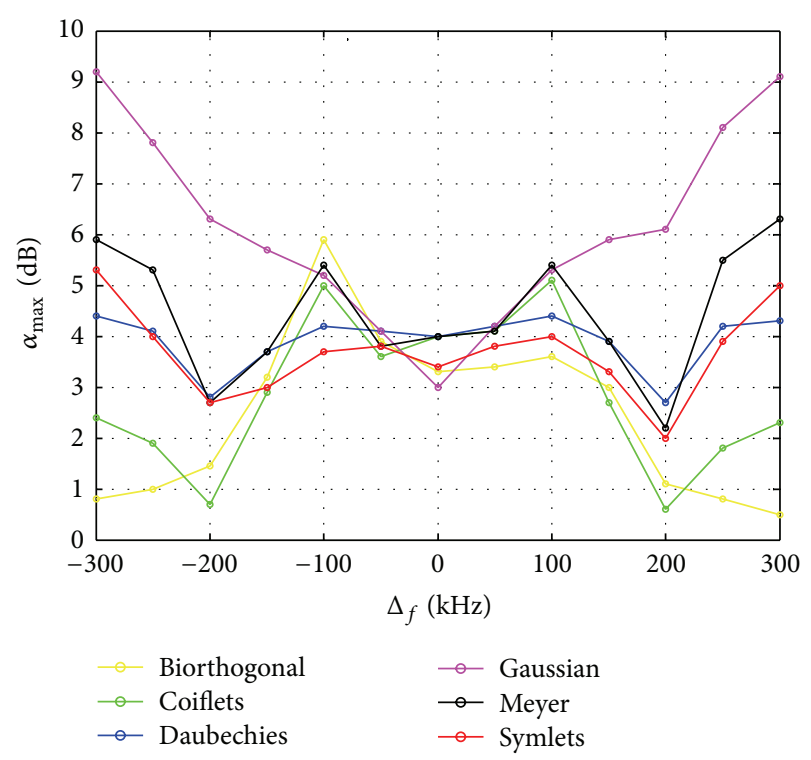

FIGURE 17: Acquisition metric $\alpha_{\max }$ with respect to the frequency offset $\Delta_{f}$ between the interference carrier and the GNSS signal carrier for different wavelet families: 7 WPD stages.

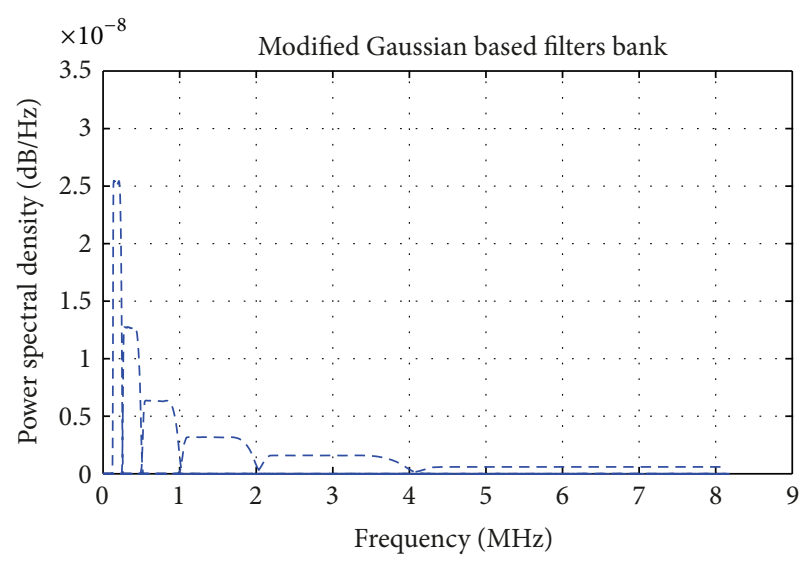

FIGURE 18: Modified Gaussian wavelet filters transfer functions.

the ADC output has been achieved through an iterative filtering process exploiting filter response derived by the Meyer wavelet family. Several other wavelet functions exist, and most of them are discussed in [35]. Figure 17 shows the acquisition metrics $\alpha_{\max }$ with respect to the frequency offset between the signal carrier and the interference carrier. Removal of the interference has been achieved for each interfered scenario exploiting 7 WPD stages and interference suppression process has been repeated using different mother 


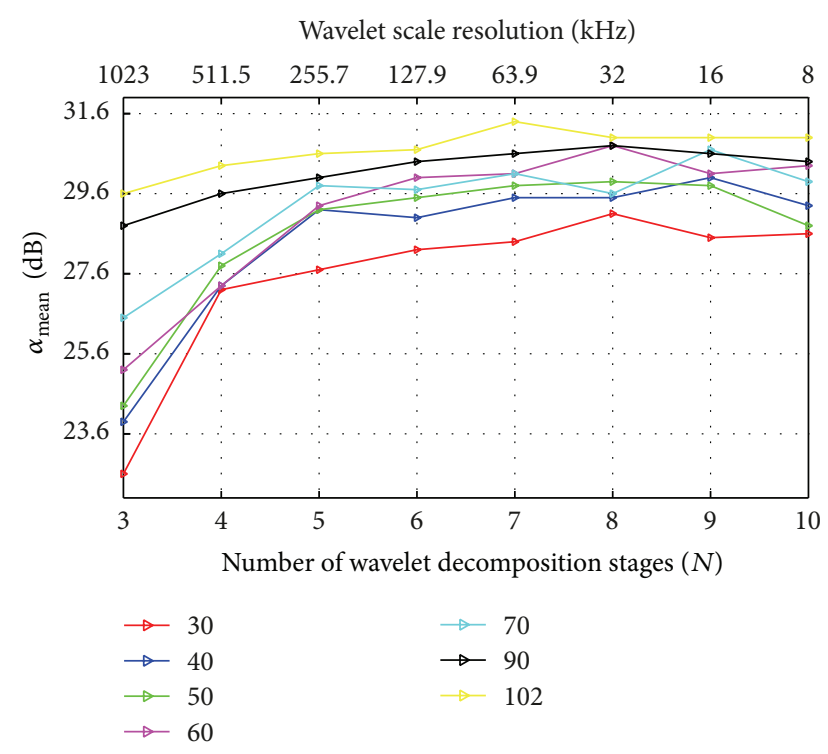

FIGURE 19: Acquisition metric $\alpha_{\text {mean }}$ with respect to the number of WPD stages $N$ and for different filter lengths of the modified Gaussian wavelet.

wavelet from which the wavelet filters transfer functions characterizing the uniform filters bank are originated.

It can be observed that, by increasing the carrier offset between the interfering signal and the GNSS signal, the acquisition metrics may not have an increasing trend. This is due to the fact that, for determined values of $\Delta_{f}$, the Narrowband interference may fall in two different subbands and thus interference removal may be negatively affected if the wavelet subbands are overlapped. For such a reason, it is important to have wavelet filter response which is selective in the frequency domain. This property is well accomplished by a wavelet function derived from an orthogonalization process of a Gaussian function: the so-called modified Gaussian function, which is described in [35]. Figure 18 shows the wavelet filters transfer functions obtained from the Gaussian wavelet function. It can be observed that such Gaussian wavelet filters are characterized by a more selective frequency response with less overlap between the subbands.

For such a reason, interference removal exploiting wavelet filters response derived from the modified wavelet Gaussian overperforms the interference process performed exploiting other wavelet function, as shown in Figure 17.

6.2.3. Wavelet Filter Length. Final investigation has been performed in order to analyse the impact of the filter length on the interference suppression. In fact, all the wavelet impulse responses are characterized by a fast decreasing amplitude in the time domain, thus allowing a good approximated implementation by means of Finite Impulse Response (FIR) filters. However, the truncation of the filter response causes a loss in the orthogonality of the subbands. The same Narrowband interference scenario has been considered, the modified Gaussian wavelet functions have been adopted for the WPD, and results are shown in Figure 19. It can be observed that by increasing the number of filter coefficients, acquisition performance improves. This is due to the fact that by increasing the wavelet filter length, wavelet function sidelobe is lowered resulting in a better orthogonality between the subbands.

6.2.4. Computational Complexity. Although Wavelet based mitigation algorithm provides high capability in interference suppression, its implementation is characterized by a non negligible complexity. Computational burden is mainly determined by the number of wavelet decomposition stages $N$ which determines the number of filtering operations according to the exponential law $2^{N}$. Furthermore, the same number of filtering operations is employed for signal reconstruction purposes. All filtering operations are realized with FIR filters with length $L$. Each output sample is obtained with $\mathrm{L}$ products and 1 single sum; thus the total number of performed operations for decomposition and reconstruction of $n$ samples of incoming signal is

$$
O(n, N, L)=2 \cdot 2^{N} \times(n L+n) .
$$

However, the filter bank implementation allows for the processing sample by sample of the incoming signal, without any signal buffering process, at the price of the delay of the decomposition stage and by the reconstruction filter bank operating on the thresholded samples. Furthermore, better efficiency of the method might be achieved by the combination of the WPD with the introduction of a frequency domain based predetection especially in those cases where interference components are not spread all over the GNSS received signal spectrum but in a determined frequency region. In such a case, a controlled dyadic scaling operation iterated on determined subbands could be implemented. It has to be remarked that wavelet decomposition is already implemented for multimedia images and audio data compression coding scheme, and the availability of Field Programmable Gate Array (FPGA) allows the WPD implementation for realtime application. Furthermore, the wavelet based algorithm can represent an efficient postprocessing technique for interference detection and characterization which can be easily implemented in potential GNSS interference monitoring stations with the aim of investigating the harsh interference environment in sensitive areas.

\section{Conclusions}

In this paper, an innovative interference mitigation algorithm exploiting the Wavelet Packet Decomposition has been presented. Detection and interference suppression mechanism has been described. Simulation results showed its effectiveness in different interference scenarios. In particular, the method is demonstrated to be effective with respect to the classical blanker in multiple pulse interference representing the DME/TACAN interference scenario on the Galileo E5a and GPS L5 frequency bands. Application of this wavelet based method showed that high interference suppression can be achieved without distorting or suppressing useful GNSS signal components, as proved by the performance at acquisition and tracking stages. Furthermore, the method 
proved to be effective also in several Narrowband interference scenarios, since it allows a tuning of the parameters to match the operating scenario. It has been shown that increasing the number of stages leads to higher interference suppression.

The final analysis confirmed also that by increasing the wavelet filters length, interference suppression performance improves thanks to the improved selectivity in frequency.

\section{Conflict of Interests}

The authors declare that there is no conflict of interests regarding the publication of this paper.

\section{References}

[1] F. Dovis, L. Musumeci, N. Linty, and M. Pini, "Recent trends in interference mitigation and spoofing detection," International Journal of Embedded and Real-Time Communication Systems, vol. 3, pp. 1-17, 2012.

[2] B. Motella, M. Pini, and F. Dovis, "Investigation on the effect of strong out-of-band signals on global navigation satellite systems receivers," GPS Solutions, vol. 12, no. 2, pp. 77-86, 2008.

[3] S. Savasta, GNSS localization techniques in interfered environment [Ph.D. dissertation], Politecnico di Torino, 2010.

[4] C. ODriscoll, M. Rao, D. Borio et al., "Compatibility analysis between LigthSquared signals and L1/E1 GNSS reception," in Proceedings of the IEEE/ION Position Location and Navigation Symposium (PLANS '12), pp. 447-454, Myrtle Beach, SC, USA, April 2012.

[5] M. D. Angelis, R. Fantacci, S. Menci, and C. Rinaldi, "An analysis of air traffic control systems interference impact on Galileo Aeronautics," in Proceedings of the 18th International Technical Meeting of the Satellite Division of The Institute of Navigation (ION GNSS '05), San Diego, Calif, USA, January 2005.

[6] L. Musumeci, J. Samson, and F. Dovis, "Experimental assessment of distance measuring equipment and tactical air navigation interference on GPS L5 and Galileo E5a frequency bands," in Proceedings of the 6th ESA Workshop on Satellite Navigation Technologies and European Workshop on GNSS Signals and Signal Processing (NAVITEC '12), pp. 1-8, Noordwijk, The Netherlands, December 2012.

[7] D. Borio, C. ODriscoll, and J. Fortuny, "GNSS jammers: effects and countermeasures," in Proceedings of the 6th ESA Workshop on Satellite Navigation Technologies and European Workshop on GNSS Signals and Signal Processing (NAVITEC '12), pp. 1-7, Noordwijk, The Netherlands, December 2012.

[8] D. Borio, "GNSS acquisition in the presence of continuous wave interference," IEEE Transactions on Aerospace and Electronic Systems, vol. 46, no. 1, pp. 47-60, 2010.

[9] A. Konovaltsev, D. S. De Lorenzo, A. Hornbostel, and P. Enge, "Mitigation of continuous and pulsed radio interference with GNSS antenna arrays," in Proceedings of the 21st International Technical Meeting of the Satellite Division of the Institute of Navigation (ION GNSS '08), pp. 1291-1300, Savannah, Ga, USA, September 2008.

[10] F. Bastide, D. Akos, C. Macabiau, and B. Roturier, "Automatic Gain Control (AGC) as an interference assessment tool," in Proceedings of the 16th International Technical Meeting of the Satellite Division of The Institute of Navigation (ION GPS/GNSS '03), pp. 2042-2053, Portland, Ore, USA, September 2003.
[11] S. Savasta, F. Dovis, R. Lesca, D. Margaria, and B. Motella, "On the interference mitigation based on ADC parameters tuning," in Proceedings of the IEEE/ION Position, Location and Navigation Symposium (PLANS '08), pp. 689-695, May 2008.

[12] F. Bastide, E. Chatre, and C. Macabiau, "GPS interference detection and identification using multicorrelator receivers," in Proceedings of the 14th International Technical Meeting of the Satellite Division of The Institute of Navigation (ION GPS '01), pp. 872-881, Salt Lake City, Utah, USA, September 2001.

[13] R. J. Erlandson, T. Kim, C. Hegarty, and A. J. van Dierendonck, "Pulsed RFI effects on aviation operations using GPS L5," in Proceedings of the National Technical Meeting of The Institute of Navigation (NTM '04), pp. 1063-1076, San Diego, Calif, USA, January 2004.

[14] C. Hegarty, A. V. Dierendonck, D. Bobyn, M. Tran, and J. Grabowski, "Suppression of pulsed interference through blanking," in Proceedings of the IAIN World Congress and the 56th Annual Meeting of The Institute of Navigation, pp. 399-408, San Diego, Calif, USA, June 2000.

[15] F. Bastide, E. Chatre, C. Macabiau, and B. Roturier, "GPS L5 and GALILEO E5a/E5b signal-to-noise density ratio degradation due to DME/TACAN signals: simulations and theoretical derivation," in Proceedings of the National Technical Meeting of The Institute of Navigation (NTM '04), pp. 1049-1062, San Diego, Calif, USA, January 2004.

[16] G. X. Gao, "DME/TACAN interference and its mitigation in L5/E5 bands," in Proceedings of the 20th International Technical Meeting of the Satellite Division of The Institute of Navigation (ION GNSS '07), pp. 1191-1200, Fort Worth, Tex, USA, September 2007.

[17] M. Raimondi, O. Julien, C. Macabiau, and F. Bastide, "Mitigating pulsed interference using frequency domain adaptive filtering," in Proceedings of the 19th International Technical Meeting of the Satellite Division of The Institute of Navigation (ION GNSS '06), pp. 2251-2260, Fort Worth, Tex, USA, September 2006.

[18] S. Savasta, L. L. Presti, and M. Rao, "Interference mitigation in GNSS receivers by a time-frequency approach," in Proceedings of the IEEE Transactions on Aerospace and Electronic Systems, vol. 49, no. 1, pp. 415-438, January 2013.

[19] E. Anyaegbu, G. Brodin, J. Cooper, E. Aguado, and S. Boussakta, "An integrated pulsed interference mitigation for GNSS receivers," Journal of Navigation, vol. 61, no. 2, pp. 239-255, 2008.

[20] M. Paonni, J. G. Jang, B. Eissfeller et al., "Innovative interference mitigation approaches: analytical analysis, implementation and validation," in Proceedings of the 5th ESA Workshop on Satellite Navigation Technologies and European Workshop on GNSS Signals and Signal Processing (NAVITEC '10), Noordwijk, The Netherlands, December 2010.

[21] M. Elhabiby, A. El-Ghazouly, and N. El-Sheimy, "A new waveletbased multipath mitigation technique," in Proceedings of the 21st International Technical Meeting of the Satellite Division of the Institute of Navigation (ION GNSS '08), pp. 885-891, Savannah, Ga, USA, September 2008.

[22] Y. Xiong, X. L. Ding, W. J. Dai, W. Chen, and D. Huang, "Mitigation of multipath effects based on GPS phase frequency feature analysis for deformation monitoring applications," in Proceedings of the 17th International Technical Meeting of the Satellite Division of the Institute of Navigation (ION GNSS '04), pp. 268276, Long Beach, Calif, USA, September 2004.

[23] X. Linyuan and L. Jingnan, "Approach for multipath reduction using wavelet algorithm," in Proceedings of the 14th International 
Technical Meeting of the Satellite Division of The Institute of Navigation (ION GPS '01), Salt Lake City, Utah, USA, September 2001.

[24] A. El-Ghazouly, "The aid of wavelets correlator in carrier phase multipath reduction and motion detection," in Proceedings of the 22nd International Technical Meeting of the Satellite Division of the Institute of Navigation (ION GNSS '09), pp. 1142-1149, Savannah, Ga, USA, September 2009.

[25] E. M. De Souza, "Multipath reduction from GPS double differences using wavelets: how far can we go?" in Proceedings of the 17th International Technical Meeting of the Satellite Division of the Institute of Navigation (ION GNSS '04), pp. 2563-2571, Long Beach, Calif, USA, September 2004.

[26] M. Elhabiby, A. El-Ghazouly, and N. El-Sheimy, "Singularity detection technique for GPS cycle slip in wavelets domain," in Proceedings of the 22nd International Technical Meeting of the Satellite Division of the Institute of Navigation (ION GNSS '09), pp. 2371-2379, Savannah, Ga, USA, September 2009.

[27] J. Wang, J. Wang, and C. Roberts, "Reducing carrier phase errors with EMD-Wavelet for precise GPS positioning," in Proceedings of the Institute of Navigation National Technical Meeting (NTM '07), pp. 919-928, San Diego, Calif, USA, January 2007.

[28] S. M. Martin, J. J. Dawkins, W. E. Travis, and D. M. Bevly, “Terrain characterization and feature extraction for automated convoys," in Proceedings of the 23rd International Technical Meeting of the Satellite Division of the Institute of Navigation (ION GNSS '10), pp. 256-265, Portland, Ore, USA, September 2010.

[29] P. Vaydianathan, Multirate Systems and Filter Banks, Signal Processing Series, Prentice-Hall, 1993.

[30] S. G. Mallat, "Theory for multiresolution signal decomposition: the wavelet representation," IEEE Transactions on Pattern Analysis and Machine Intelligence, vol. 11, no. 7, pp. 674-693, 1989.

[31] M. Fantino, M. Nicola, and A. Molino, "N-Gene GNSS receiver: benefits of software radio in navigation," in Proceedings of the European Navigation Conference (ENC-GNSS '09), Naples, Italy, May 2009.

[32] J. Soubielle, W. Vigneau, J. Samson, D. J. Banos, and L. Musumeci, "Description of an Interference Test Facility (ITF) to assess GNSS receivers performance in presence of interference," in Proceedings of the 5th ESA Workshop on Satellite Navigation Technologies and European Workshop on GNSS Signals and Signal Processing (NAVITEC '10), December 2010.

[33] D. Margaria, E. Falletti, B. Motella, M. Pini, and G. Povero, "NFUELS, a GNSS educational tool for simulation and analysis of a variety of signal in space," in Proceedings of the European Navigation Conference (ENC-GNSS '10), Braunschweig, Germany, October 2010.

[34] L. Musumeci and F. Dovis, "Performance assessment of wavelet based technique in mitigating narrow-band interference," in Proceedings of the International Conference on Localization and GNSS (ICL-GNSS '13), pp. 1-6, Turin, Italy, June 2013.

[35] F. Dovis, Wavelet based designed of digital multichannel communications systems [Ph.D. dissertation], Politecnico di Torino, 1999. 

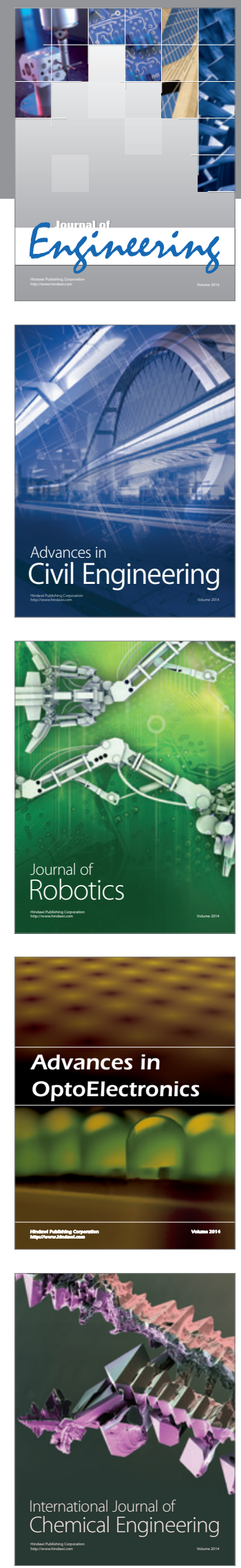

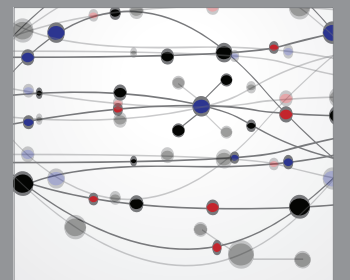

The Scientific World Journal
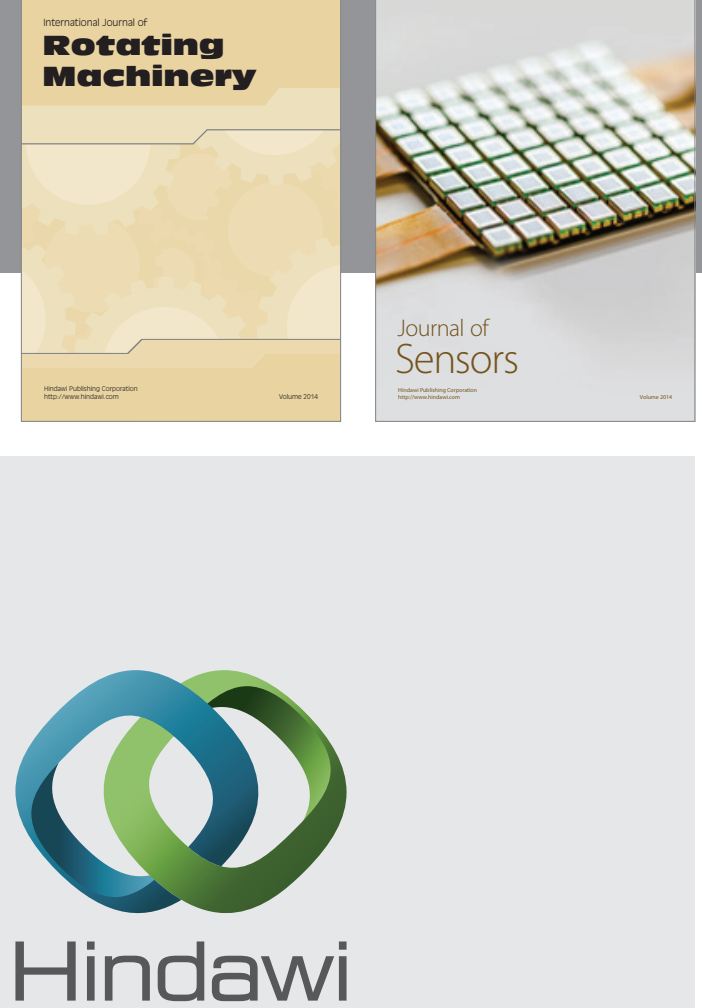

Submit your manuscripts at http://www.hindawi.com
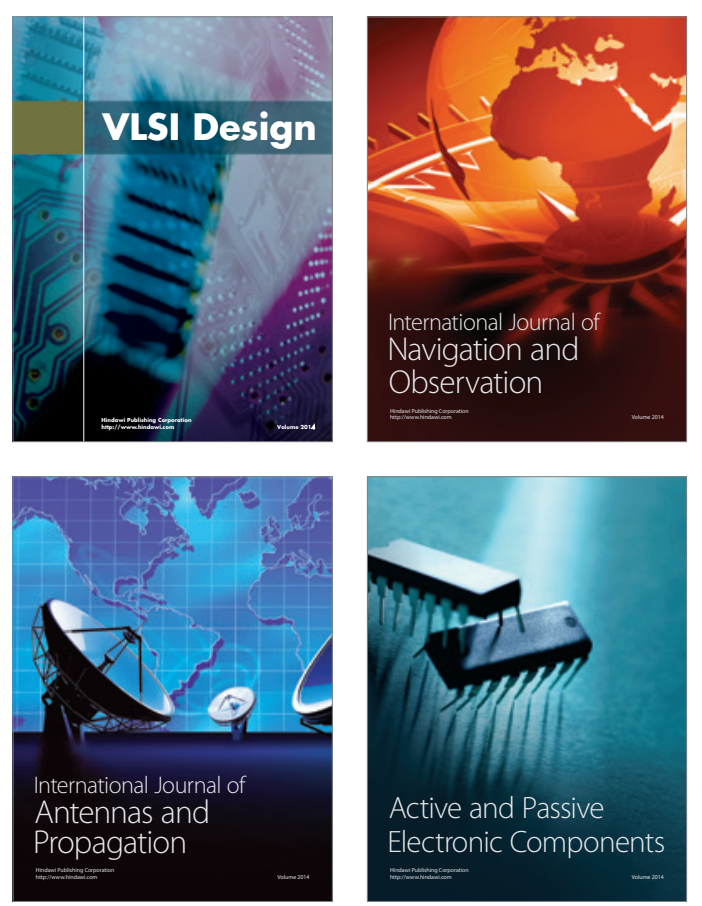
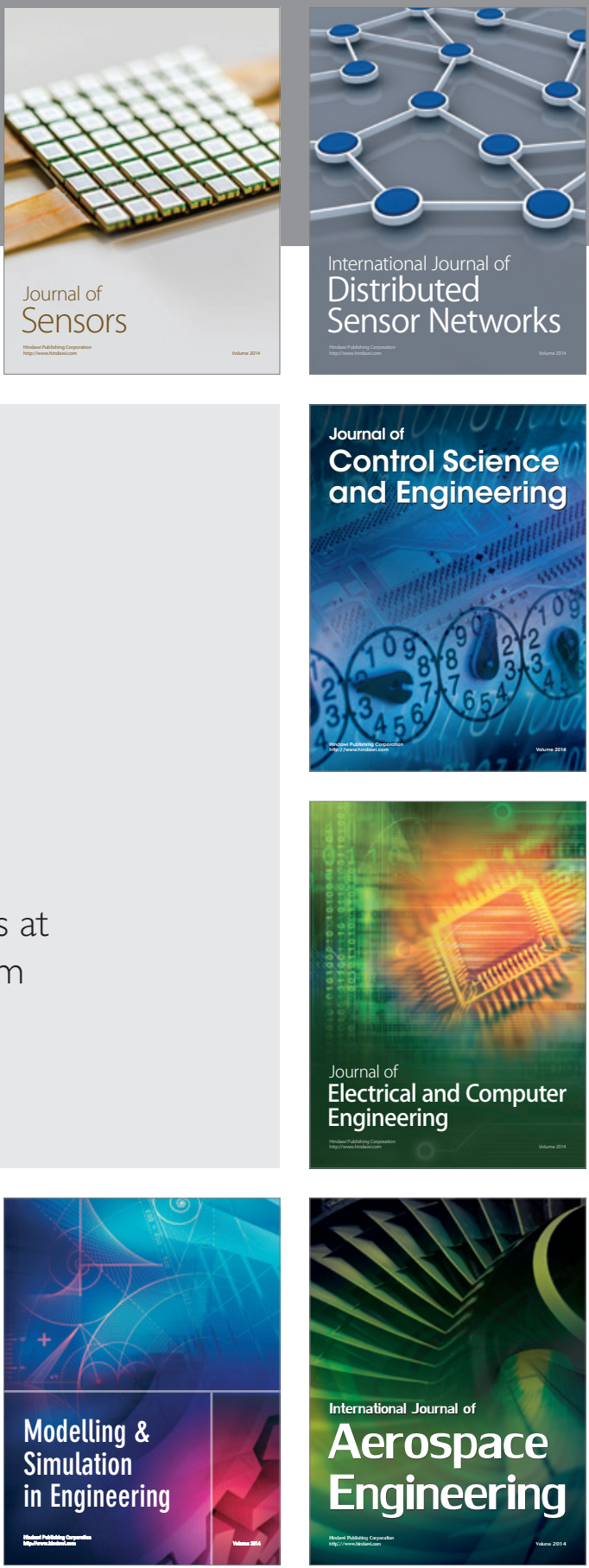

Journal of

Control Science

and Engineering
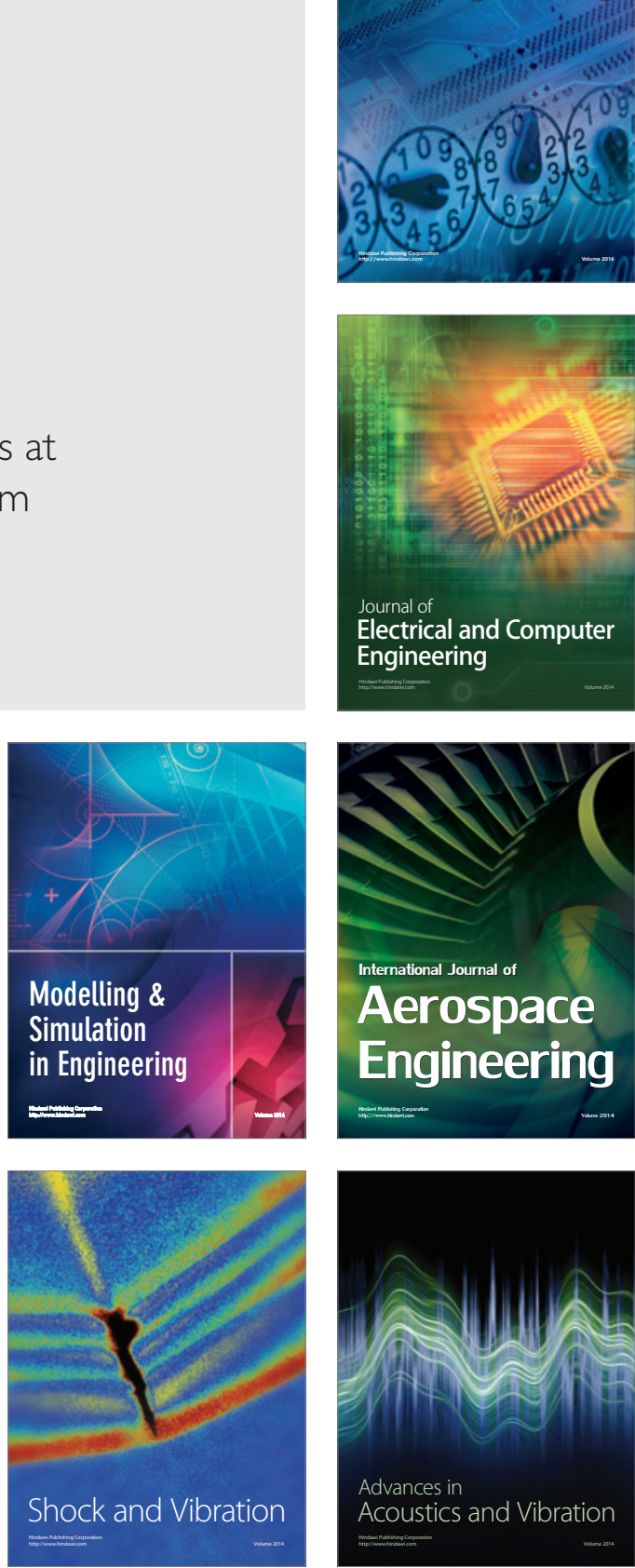